\title{
Value of transrectal contrast-enhanced ultrasound with clinical indicators in the prediction of bone metastasis in prostate cancer
}

\author{
Hua Hong ${ }^{1 \#}$, Danyan Liang ${ }^{1 \#}$, Qian Liu $^{1}$, Guozhu Wu ${ }^{1}$, Ran Sun ${ }^{1}$ Juzhen Liu ${ }^{2}$, Feng Wang ${ }^{3}$, Fang Wang ${ }^{1}$ \\ ${ }^{1}$ Department of Ultrasonography, Inner Mongolia People's Hospital, Hohhot, China; ${ }^{2}$ Department of Nuclear Medicine, Inner Mongolia People’s \\ Hospital, Hohhot, China; ${ }^{3}$ Department of Pathology, Inner Mongolia People's Hospital, Hohhot, China
}

\begin{abstract}
Contributions: (I) Conception and design: H Hong; (II) Administrative support: H Hong; (III) Provision of study materials or patients: Q Liu, G Wu, R Sun, J Liu, Feng Wang, Fang Wang; (IV) Collection and assembly of data: Q Liu, G Wu, R Sun, J Liu, Feng Wang, Fang Wang; (V) Data analysis and interpretation: D Liang; (VI) Manuscript writing: All authors; (VII) Final approval of manuscript: All authors.
\end{abstract}

"These authors contributed equally to this work.

Correspondence to: Hua Hong, PhD. Department of Ultrasonography, Inner Mongolia People's Hospital, Hohhot, China. Email: 6622306hong@163.com.

Background: Transrectal contrast-enhanced ultrasound is an examination that can be used to diagnose and characterize prostate cancer by displaying tissue blood perfusion. To explore the value of transrectal contrastenhanced ultrasound combined with clinical factors in predicting prostate cancer bone metastasis.

Methods: We retrospectively analyzed transrectal contrast-enhanced ultrasound examination data, imaging examination data [single-photon emission computed tomography (SPECT)/computed tomography (CT), CT, magnetic resonance imaging (MRI), and/or bone scan], clinical laboratory data, and pathological Gleason score of 163 patients with prostate cancer. They were randomly divided into the modeling and validation data sets. A model for predicting prostate cancer bone metastasis was established by logistic regression in the modeling data set. The differentiation, consistency, and benefits of the model were verified using the validation data set. A nomogram of the prediction model for bone metastasis of prostate cancer was drawn.

Results: Among 163 patients with prostate cancer, 65 had bone metastasis. Total prostate-specific antigen, alkaline phosphatase, and the transrectal contrast-enhanced ultrasound parameter area under the curve were independently associated with prostate cancer bone metastasis, with OR values of $2.845,2.839$, and 1.004 , respectively. The area under the receiver operating characteristic curve of the prostate cancer bone metastasis prediction model was 0.804 . In the training set, using a cutoff of 0.659 , sensitivity was $52.8 \%$, and specificity was $95.7 \%$. In the validation set, using a cutoff of 0.659 , sensitivity was $58.6 \%$, and specificity was $98.1 \%$. The area under the curve of the validation set was 0.799 . The Hosmer-Lemeshow goodness-of-fit test showed that the calibration ability of the validation set was not statistically different from the training set $(\mathrm{P}=0.136)$. The decision curve analysis showed that the model had high benefits.

Conclusions: The nomogram that includes the transrectal contrast-enhanced ultrasound parameter area under the curve and the clinical parameters total prostate-specific antigen, and alkaline phosphatase can be used to personalize the risk of prostate cancer bone metastases.

Keywords: Prostate cancer (PCa); bone metastasis; transrectal contrast-enhanced ultrasound (TR-CEUS); total prostate-specific antigen (TPSA); alkaline phosphatase (ALP)

Submitted Apr 06, 2021. Accepted for publication Oct 29, 2021.

doi: 10.21037/qims-21-365

View this article at: https://dx.doi.org/10.21037/qims-21-365 


\section{Introduction}

Prostate cancer $(\mathrm{PCa})$ is a common malignant tumor in males in western countries. In 2018, there were 1,276,106 new cases of PCa and 358,989 deaths in the world, ranking $3^{\text {rd }}$ in incidence $(1,2)$. In China, the incidence of $\mathrm{PCa}$ is 60.3 per 100,000 , and the mortality rate is 26.6 per 100,000 ; it ranks $7^{\text {th }}$ in the incidence of male malignancies and 10th in the mortality rate (3). The most important cause of death in PCa is distant metastasis, causing organ failure or loss of function, leading to death. The most common distant metastatic site is bone. There is a lack of typical clinical manifestations of PCa in the early stage. Most patients with PCa are diagnosed with advanced stage and already have bone metastasis (4). The 5 -year survival rate of $\mathrm{PCa}$ patients with bone metastases is only $25 \%$, while the median survival time is 40 months (5). Therefore, predicting PCa bone metastasis and conducting clinical intervention earlier should reduce the mortality of patients with $\mathrm{PCa}$ and improve their quality of life.

At present, the clinical imaging studies for PCa bone metastasis are mostly focused on diagnosing and staging the lesions $(6,7)$ and rely on positron emission tomography $(8,9)$, which is not widely available in some areas of the world. The prediction of bone metastasis is mainly based on clinical, biochemical, and tumor markers $(10,11)$. Transrectal contrast-enhanced ultrasound (TR-CEUS) is an examination that can be used to diagnose and characterize PCa $(12,13)$. TR-CEUS is based on tumor neovascularization, a necessary condition for the formation, survival, and invasion of $\mathrm{PCa}$ $(14,15)$. Through the nonlinear effect of microbubble vibration, TR-CEUS increases the signal-to-noise ratio, enables real-time dynamic observation of tissue perfusion, improves microvascular imaging of prostate tumors, and can distinguish tumor lesions from normal tissues, thus improving the detection rate of $\mathrm{PCa}$ (16).

The occurrence of bone metastasis affects the choice of treatment for PCa. Identifying "high-risk" patients for bone metastases may allow patients to benefit from targeted therapy. The use of TR-CEUS parameters and clinical indicators to predict $\mathrm{PCa}$ with bone metastasis has rarely been reported. Therefore, this study explored the value of TR-CEUS combined with clinical factors in predicting PCa bone metastasis.

\section{Methods}

\section{Study design and patients}

This retrospective study was performed at the Department of Ultrasound Medicine of the Inner Mongolia People's Hospital and included patients with PCa diagnosed between March 2016 and March 2019. The study was conducted in accordance with the Declaration of Helsinki (as revised in 2013). The study was approved by the ethics committee of the Inner Mongolia Autonomous Region People's Hospital, and individual consent for this retrospective analysis was waived.

The inclusion criteria were (I) suspected for PCa based on digital rectal examination; (II) total prostatespecific antigen (TPSA) $\geq 4 \mathrm{ng} / \mathrm{mL}$; (III) underwent TRCEUS; (IV) underwent single-photon emission computed tomography (SPECT)/computed tomography (CT), CT, magnetic resonance imaging (MRI), and/or bone scan; and $(\mathrm{V})$ complete clinical and biochemical data available, including age, serum TPSA, alkaline phosphatase (ALP), and pathological Gleason score. The exclusion criteria were (I) non-PCa patients; (II) incomplete clinical information; (III) lack of complete laboratory tests; (IV) incomplete pathological data; or (V) lack of CEUS, SPECT/CT, CT, MRI, and/or bone scan.

The diagnostic criteria for bone metastasis were (I) patients diagnosed with PCa; (II) exclusion of bone metastases caused by other malignant tumors or primary malignant tumors of bone; (III) bone metastases were found by two or more imaging methods based on the comprehensive judgment of experienced imaging physicians based on the patient bone scan, SPECT/CT, CT, MRI, and other imaging examinations; and (IV) bone scan, SPECT/ CT, CT, and/or MRI follow-up of the suspicious lesion interval showed significant progression.

\section{Transrectal ultrasound (TRUS) examination}

An IU22-G4 ultrasound system (Philips, Best, The Netherlands) equipped with a $5-9-\mathrm{MHz}$ transrectal endscan probe with contrast function and QLAB analysis software (IU22-G4, Philips, Best, The Netherlands) were used for TR-CEUS. The prostate gland and peripheral area were thoroughly explored to see if the prostate capsule was intact, whether there were echogenic abnormalities, solid nodules, and check the blood flow distribution in the prostate and nodules.

Injectable sulfur hexafluoride $\left(\mathrm{SF}_{6}\right)$ microbubbles (SonoVue, Bracco, Italy) were used. If there were no abnormalities in the conventional prostate TRUS, the largest transverse section was selected as the contrast observation level. If there were an abnormal echo zone 
(nodule) and/or a rich blood supply layer in the prostate, the level of the abnormal echo zone (nodule) and/or the maximum cross-section of the blood supply was taken as the contrast observation level. After the probe was placed in the rectum and the prostate contrast plane was selected, $2.4 \mathrm{~mL}$ of SonoVue suspension was quickly injected into the elbow vein $(t=0)$. The timing button of the contrast software was started to record the time. Then, $5 \mathrm{~mL}$ of $0.9 \%$ sodium chloride solution was quickly injected, and the microbubbles of the contrast plane were dynamically observed while the injection was being performed. During the perfusion process, the dynamic image length of each layer was $120 \mathrm{~s}$. When the CEUS was completed, the recorded images were stored on the machine's hard drive. When performing the CEUS, the planes with the most abundant blood supply and/or abnormal nodules were selected as the observation planes during the CEUS examination. When the planes with the most abundant blood supply and/or abnormal nodules were not in the same plane, a second contrast agent injection was required to observe the two planes. When the intra-prostate contrast microbubble echo disappeared, the next contrast injection and contrast examination were performed following the same procedure. During CEUS, the sound pressure was adjusted to $50 \mathrm{~dB}$, the mechanical index was 0.07 , the dynamic range was $65 \mathrm{~dB}$, and the depth was $50 \mathrm{~mm}$. The focus was set at the bottom of the image, and the instrument parameters of all patients were consistent.

\section{Image analysis}

Two CEUS-experienced sonographers used the QLAB contrast-enhanced ultrasonography software to analyze the CEUS data by replaying the CEUS process and analyzing the contrast's perfusion characteristics and perfusion mode agent in the prostate. The analysis included selecting the target region, plotting the time-intensity analysis curve, and recording the peak intensity (PI), time to peak (TTP), rise time (RT), mean transit time (MTT), and area under the curve (AUC), wash-in slope (WIS), and intensity from peak to one-half time (HT). The region of interest (ROI) area was divided differently for each patient and selected according to the analysis of CEUS by two experienced sonographers. If there was an area of abnormal enhanced nodules, it was selected as the ROI area. In the case of diffuse enhancement or heterogeneous enhancement, the region with the most significant contrast enhancement was selected as the ROI area.

\section{Biopsy}

A Magnum automatic biopsy gun and disposable biopsy needle (model 18-20, a needle length of $20 \mathrm{~cm}$ ) (Bard Biopsy Systems, Tempe, AZ, USA). Gleason's score was analyzed by pathologists. According to the 2016 WHO Prostate cancer Classification Guidelines (17), Gleason score was divided into five grades: Grade 1: Gleason score $\leq 6$; Grade 2: Gleason score 3+4 =7; Grade 3: Gleason score 4+3=7; Level 4: Gleason score 8 (including Gleason 3+5, Gleason $5+3$ and Gleason 4+4); Level 5: Gleason scores 9 and 10 (including Gleason 4+5, Gleason 5+4 and Gleason 5+5).

\section{Patient screening process}

A total of 364 patients, whose serum TPSA levels were continuously $\geq 4 \mathrm{ng} / \mathrm{mL}$ and were suspected with PCa by clinical urologists, underwent TR-CEUS and prostate biopsy. Among them, 166 patients were pathologically confirmed with PCa (Figure 1). Except for three patients who gave up treatment, the remaining 163 patients underwent SPECT/CT, CT, and/or MRI and were included in the study. There were 65 patients with bone metastases and 98 without. The serum ALP and other clinical indicators were collected. The 163 patients were randomly divided into the training $(\mathrm{n}=82)$ and validation $(\mathrm{n}=81)$ sets.

\section{Statistical analysis}

The continuous variables were presented as means \pm standard deviation or medians (ranges) and analyzed using the Mann-Whitney U-test or Student's $t$-test, according to the Kolmogorov-Smirnov test for normal distribution. The categorical variables were presented using $\mathrm{n}(\%)$ and were analyzed using Pearson's chi-square test. Univariable meaningful variables were included in the logistic regression analysis to identify factors associated with bone metastasis; those with a $P$ value $\leq 0.05$ were included in the multivariable model. To construct a well-calibrated and discriminative nomogram for predicting bone metastasis, a model was developed in a training set and then validated in a validation dataset. SPSS 16.0 (IBM, Armonk, NY, USA) was used to generate a random number table to randomize the patients 1:1 between the two sets. The 163 patients were randomly divided into the training $(\mathrm{n}=82)$ and validation $(\mathrm{n}=81)$ sets. A logistic regression model was used to construct the nomogram. In logistic regression, TPSA, ALP, and Gleason 


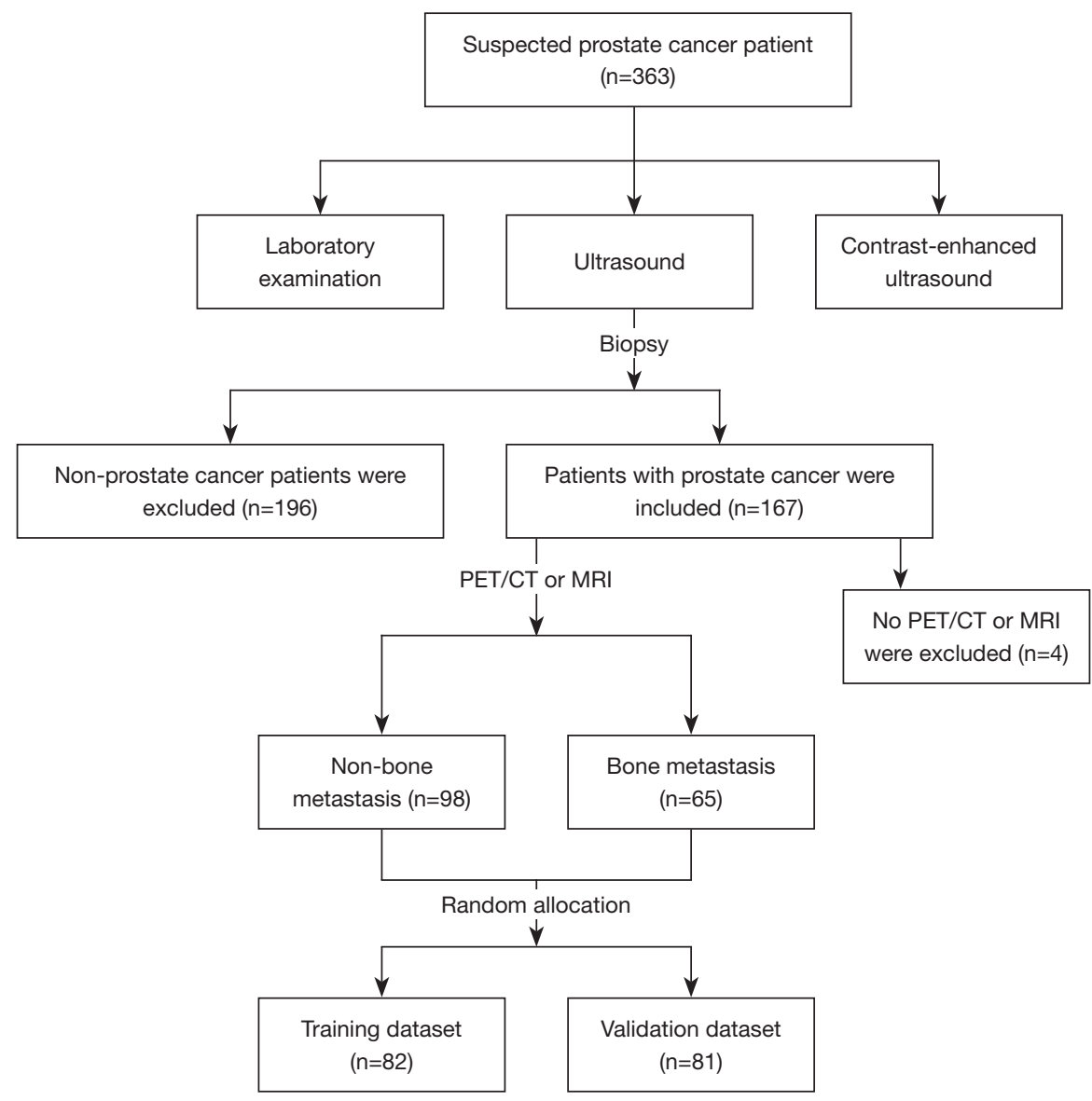

Figure 1 Study flowchart. PET, positron emission tomography; CT, computed tomography; MRI, magnetic resonance imaging.

Table 1 Variable assignment table

\begin{tabular}{ll}
\hline Variable & Values \\
\hline Bone metastasis & No bone metastasis $=0 ;$ bone metastasis $=1$ \\
TPSA & $<20 \mathrm{ng} / \mathrm{mL}=1 ; 20-30 \mathrm{ng} / \mathrm{mL}=2 ;>30 \mathrm{ng} / \mathrm{mL}=3$ \\
ALP & $<100 \mathrm{U} / \mathrm{L}=1 ; 100-150 \mathrm{U} / \mathrm{L}=2 ;>150 \mathrm{U} / \mathrm{L}=3$ \\
Gleason score & $\leq 6=1 ; 3+4=2 ; 4+3=3 ; 8=4 ; 9-10=5$ \\
\hline
\end{tabular}

TPSA, total prostate-specific antigen; ALP, alkaline phosphatase.

grades were analyzed according to ordinal data, and other variables were analyzed according to continuous data (Table 1). Nomogram performance was quantified with respect to discrimination and calibration. Discrimination (the ability of a nomogram to separate patients with bone metastasis) was quantified using the area under the receiver operating characteristic (ROC) curve (AUC). Calibration was assessed graphically by plotting the relationship between actual probabilities and predicted probabilities using the Hosmer goodness-of-fit test. Calibration curves were also drawn to evaluate the concordance between predicted and observed probabilities. Decision curve analyses (DCA) were used to analyze the benefits performance. Comparative analysis of the diagnostic value of the model and single index was conducted according to Hanley \& McNeil (18).

All tests were two-sided, and $\mathrm{P} \leq 0.05$ was deemed significant. Statistical analyses were conducted using Stata (StataCorp LP, College Station, TX, USA) and the R 
Table 2 Clinical symptoms of the patients

\begin{tabular}{|c|c|c|c|c|c|c|c|}
\hline Symptoms & \multicolumn{2}{|c|}{ Training set } & \multicolumn{2}{|c|}{ Validation dataset } & \multicolumn{3}{|c|}{ All } \\
\hline Dysuria & $36(78.3)$ & $24(66.7)$ & $40(76.9)$ & $23(79.3)$ & $76(77.6)$ & $47(72.3)$ & 0.446 \\
\hline Hematuresis & $6(13.0)$ & 0 & $6(11.5)$ & $2(6.9)$ & $12(12.2)$ & $2(3.1)$ & 0.048 \\
\hline Frequency and urgency of urination & $22(47.8)$ & $12(33.3)$ & $24(46.2)$ & $10(34.5)$ & $46(46.9)$ & $22(33.8)$ & 0.097 \\
\hline Bone pain & $3(6.5)$ & $5(13.9)$ & $2(3.8)$ & $12(41.4)$ & $5(5.1)$ & $17(26.2)$ & $<0.001$ \\
\hline
\end{tabular}

All data are shown as $n(\%)$.

programming language and environment version 3.4.1 (http://cran.r-project.org).

\section{Results}

\section{Characteristics of the patients}

Among the 163 patients with PCa, 39.9\% (65/163) had bone metastasis, and 60.1\% (98/163) had no bone metastasis. In the training set, there were 36 cases of bone metastasis and 46 cases of no bone metastasis. In the validation set, 52 patients had bone metastasis, and 29 had no bone metastasis (Figure 1). Among the patients with bone metastasis, $72.3 \%$ showed dysuria, $3.1 \%$ showed hematuria, $10.8 \%$ showed urinary retention, $33.8 \%$ showed frequency and urgency of urination, and $26.2 \%$ reported bone pain. The proportion of hematuria in patients with bone metastasis was lower than in patients without bone metastasis, while the proportion of bone pain in patients with bone metastasis was higher than in patients without bone metastasis $(\mathrm{P}<0.05)$, while there was no significant difference in other symptoms between patients with and without bone metastasis. The specific clinical symptoms of the patients are shown in Table 2 .

There were no significant differences in age and prostate volume between the two groups. Bone metastasis was associated with higher TPSA, ALP, and Gleason grades (Table 3). Table S1 shows the characteristics of the patients in the two sets.

\section{TR-CEUS}

The TR-CEUS parameters PI and AUC of patients with $\mathrm{PCa}$ with bone metastases were higher than in those without bone metastases, while the other parameters were not significantly different (Table 4).

\section{Multivariable regression logistic analysis}

The multivariable analysis showed that compared with TPSA $<20 \mathrm{ng} / \mathrm{mL}$, the risk of PCa bone metastases increased by 1.845 times for each $10-\mathrm{ng} / \mathrm{mL}$ increase in TPSA (because the OR value was 2.845 , the increased risk is $\mathrm{OR}-1=1.845$ times). Compared with ALP $<100 \mathrm{U} / \mathrm{L}$, each 50-U/L increase in ALP increased the risk of $\mathrm{PCa}$ bone metastases by 1.839 times (because the OR value was 2.839, the increased risk is 1.839 times). For each AUC unit increase, the risk of bone metastasis increased by 0.004 times in PCa patients (because the OR value was 1.004 , the increased risk is 0.004 times) (Table 5).

\section{Nomogram}

Figure 2 shows the nomogram predicting bone metastasis in patients with PCa. The top row shows the point assignment for each variable. Rows 2-4 indicate the variables included in the nomogram. For an individual patient, each variable is assigned a point value based on the characteristics. The points assigned to each of the three variables are summed, and the total points are indicated in row 5 .

\section{Evaluation of the predictive model}

The degree of discrimination of the model was evaluated using a ROC curve. In the training sample, the AUC of the model was 0.804 (Figure $3 A$ ). In the validation sample, the AUC of the model was 0.799 (Figure 3B), which has a high 
Table 3 Characteristics of the patients

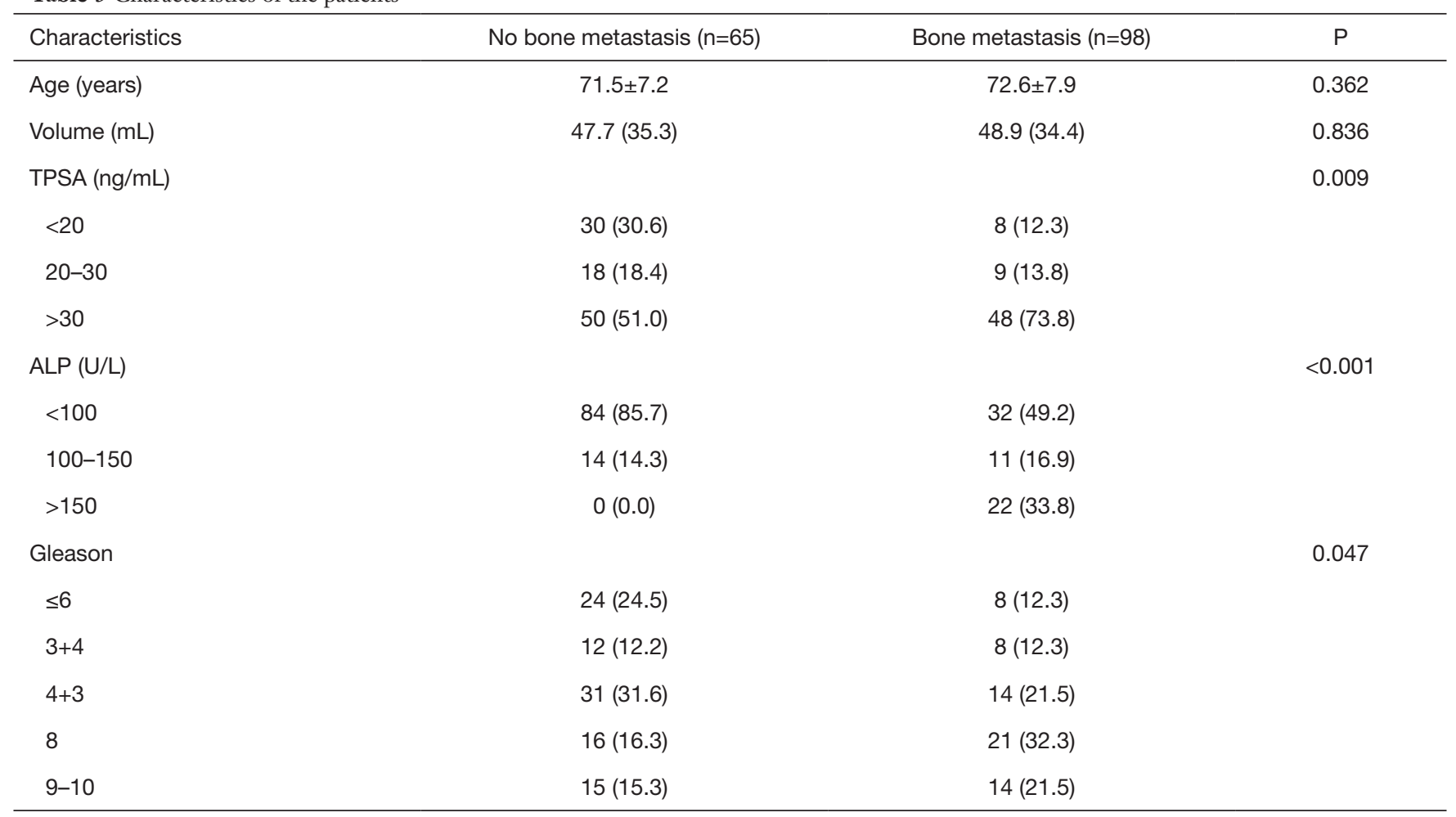

Continuous data are presented as mean \pm standard deviation or median (interquartile range). Categorical data are shown as $n$ (\%). TPSA, total prostate-specific antigen; ALP, alkaline phosphatase.

Table 4 Contrast-enhanced ultrasound parameters

\begin{tabular}{lccc}
\hline Parameters & Non-bone metastasis & Bone metastasis & P \\
\hline PI (dB) & $7.30(3.00)$ & $7.88(4.72)$ & $27.39(13.07)$ \\
MTT (s) & $25.67(13.50)$ & $433.07(308.92)$ & 0.022 \\
AUC (dBs) & $403.95(200.32)$ & $40.51(20.83)$ & 0.003 \\
HT (s) & $36.80(14.90)$ & $0.91(0.92)$ & 0.427 \\
WIS (s) & $1.38(0.94)$ & $24.84(8.84)$ & 0.150 \\
TTP (s) & $24.20(8.97)$ & $6.96(3.88)$ & 0.891 \\
RT (s) & $6.54(3.30)$ & 0.197 &
\end{tabular}

All data are shown as median (interquartile range). PI, peak intensity; MTT, mean transit time; AUC, area under the curve; HT, intensity from peak to one-half time; WIS, wash-in slope; TTP, time to peak; RT, rise time.

degree of discrimination. In the training dataset, using a cut-off value of 0.659 , sensitivity was $52.8 \%$, and specificity was $95.7 \%$. In the validation dataset, using a cut-off value of 0.659 , sensitivity was $58.6 \%$, and specificity was $98.1 \%$. The results showed that the predictive value of the bone metastasis model was higher than that of the single index.
The results of the predictive value of the single indicators are shown in Table S2.

The validation dataset was evaluated using the HosmerLemeshow goodness-of-fit test to assess the predictive model's calibration ability. The validation dataset's calibration ability was not significantly different from the 
Table 5 Logistic regression to identify risk factors of bone metastases of PCa in the training dataset

\begin{tabular}{|c|c|c|c|c|c|c|}
\hline Indicators & \multicolumn{3}{|c|}{ Univariable analysis } & \multicolumn{3}{|c|}{ Multivariable analysis } \\
\hline Age & -0.013 & $0.987(0.936-1.041)$ & 0.639 & - & - & - \\
\hline Volume & 0.001 & $1.000(0.991-1.010)$ & 0.963 & - & - & - \\
\hline Gleason & 0.231 & $1.260(0.885-1.794)$ & 0.199 & - & - & - \\
\hline MTT & 0.019 & $1.019(0.979-1.061)$ & 0.348 & - & - & - \\
\hline HT & 0.011 & $1.011(0.981-1.042)$ & 0.479 & - & - & - \\
\hline WIS & 0.199 & $1.221(0.783-1.904)$ & 0.379 & - & - & - \\
\hline TTP & 0.003 & $1.003(0.952-1.057)$ & 0.906 & - & - & - \\
\hline ALP & 1.136 & $3.116(1.481-6.554)$ & 0.003 & 1.043 & $2.839(1.198-6.725)$ & 0.018 \\
\hline AUC & 0.004 & $1.004(1.001-1.006)$ & 0.003 & 0.004 & $1.004(1.001-1.007)$ & 0.007 \\
\hline Constant & - & - & - & -6.136 & - & - \\
\hline
\end{tabular}

PCa, prostate cancer; Cl, confidence interval; PI, peak intensity; MTT, mean transit time; HT, intensity from peak to one-half time; WIS, wash-in slope; TTP, time to peak; RT, rise time; TPSA, total prostate-specific antigen; ALP, alkaline phosphatase; AUC, area under the curve.

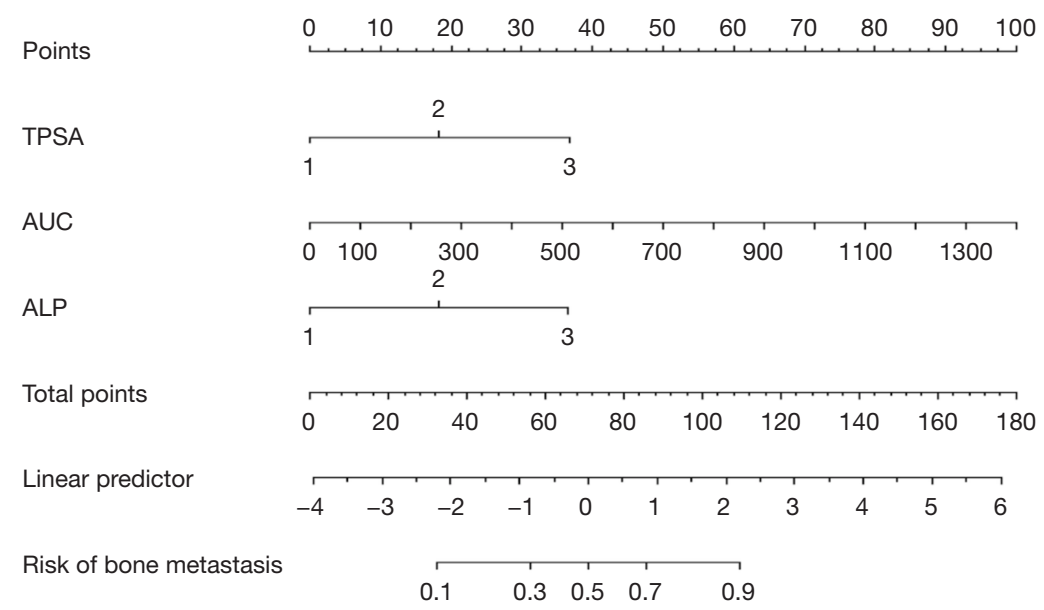

Figure 2 Nomogram predicting bone metastasis in patients with PCa. The top row shows the point assignment for each variable. Rows 2-4 indicate the variables included in the nomogram. For an individual patient, each variable is assigned a point value based on the characteristics. The points assigned to each of the three variables are summed, and the total points are indicated in row 5 . The bottom row shows the probability of the patient having bone metastasis. TPSA 1, 2, and 3 respectively represent $<20,20-30$, and $>30 \mathrm{ng} / \mathrm{mL}$. ALP 1 , 2 , and 3 respectively represent $<100,100-150$, and $>150 \mathrm{U} / \mathrm{L}$. PCa, prostate cancer; TPSA, prostate-specific antigen; AUC, area under the curve; ALP, alkaline phosphatase. 

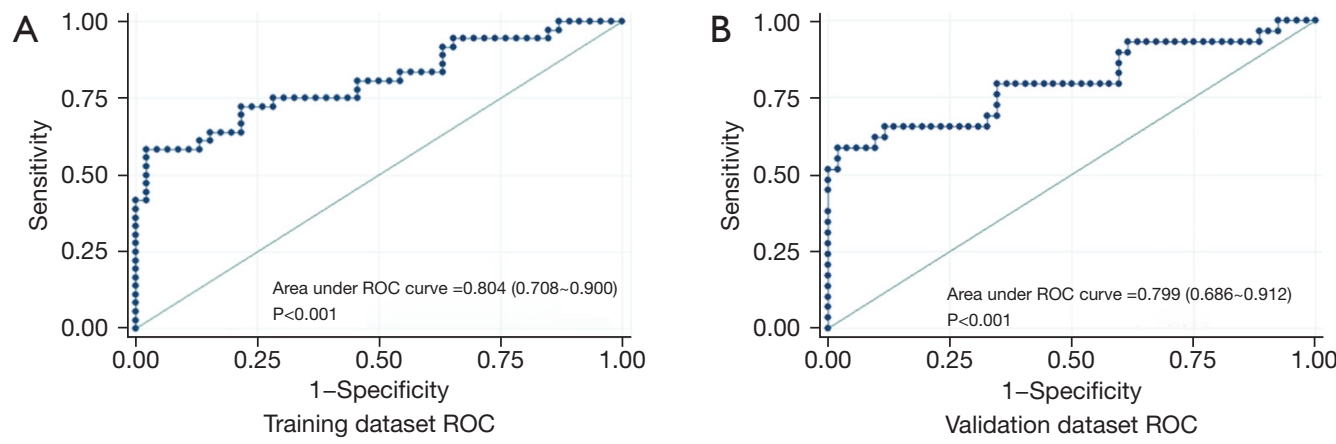

Figure 3 The receiver operating characteristics (ROC) curves of the training (A) and validation (B) datasets.
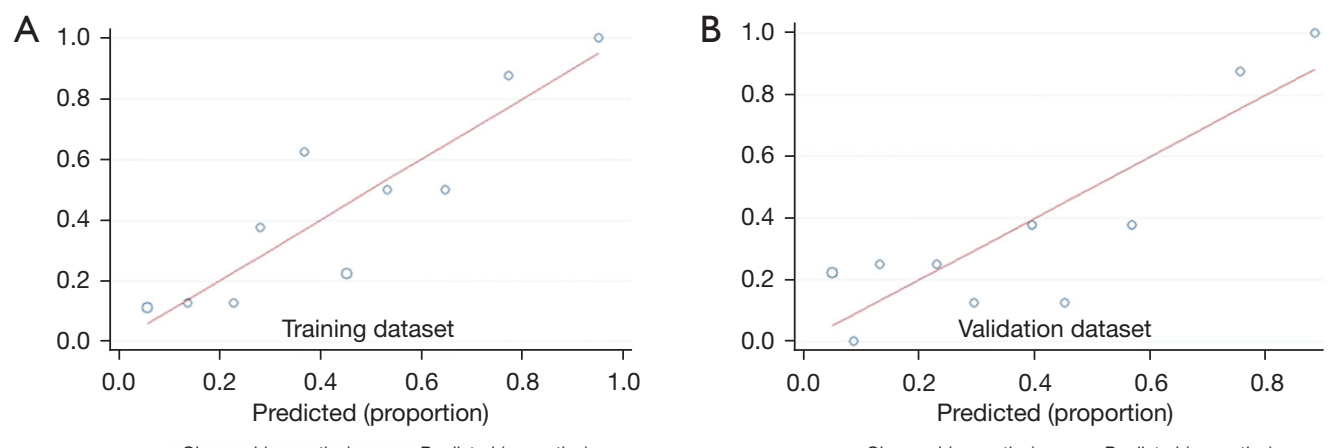

Figure 4 The calibration curve. The nomogram-predicted probability of bone metastases was plotted on the $x$-axis, and the actual bone metastases were plotted on the y-axis. Dashed lines along the 45-degree line through the point of origin represent the perfect calibration models in which the predicted probabilities are identical to the actual probabilities.

training dataset $(\mathrm{P}=0.136)$ (Figure 4). On the DCA, the nomogram showed a superior net benefit (Figure 5).

\section{Representative cases}

Two representative patients are presented in Figure S1 (low nomogram score and no bone metastasis) and Figure S2 (high nomogram score and bone metastases).

\section{Discussion}

TR-CEUS is an examination that can be used to diagnose and characterize PCa by displaying tissue blood perfusion. The use of TR-CEUS parameters and clinical indicators to predict $\mathrm{PCa}$ with bone metastasis has rarely been reported. This study aimed to explore the value of TRCEUS combined with clinical factors in predicting $\mathrm{PCa}$ bone metastasis. The results strongly suggest that the nomogram that includes the TR-CEUS parameter AUC and the clinical parameters TPSA and ALP can be used to personalize the risk of $\mathrm{PCa}$ bone metastases.

Some authors reported that TPSA could be considered an independent risk factor to evaluate the propensity for PCa to metastasize (19). This study found that when using TPSA $<20 \mathrm{ng} / \mathrm{mL}$ as a reference, the proportion of patients with $\mathrm{PCa}$ and bone metastases was increased in the patients with TPSA $20-30$ and $>30 \mathrm{ng} / \mathrm{mL}$. The multivariable analysis showed that the TPSA levels of 20-30 and $>30 \mathrm{ng} / \mathrm{mL}$ were independent risk factors for PCa with bone metastases. For each unit increase of TPSA, the risk increased by 1.845 times. TPSA can induce osteoclast precursor cell apoptosis to promote osteoblast growth and reduce osteoclasts, promoting $\mathrm{PCa}$ bone metastasis (20). The prevalence of bone metastases in PCa patients with TPSA $\geq 20 \mathrm{ng} / \mathrm{mL}$ was $14.0-26.5 \%$, and the sensitivity and specificity of TPSA $\geq 20 \mathrm{ng} / \mathrm{mL}$ for predicting bone metastasis were $86.5 \%$ and $41.2 \%$, respectively (21). In 2010, the National 

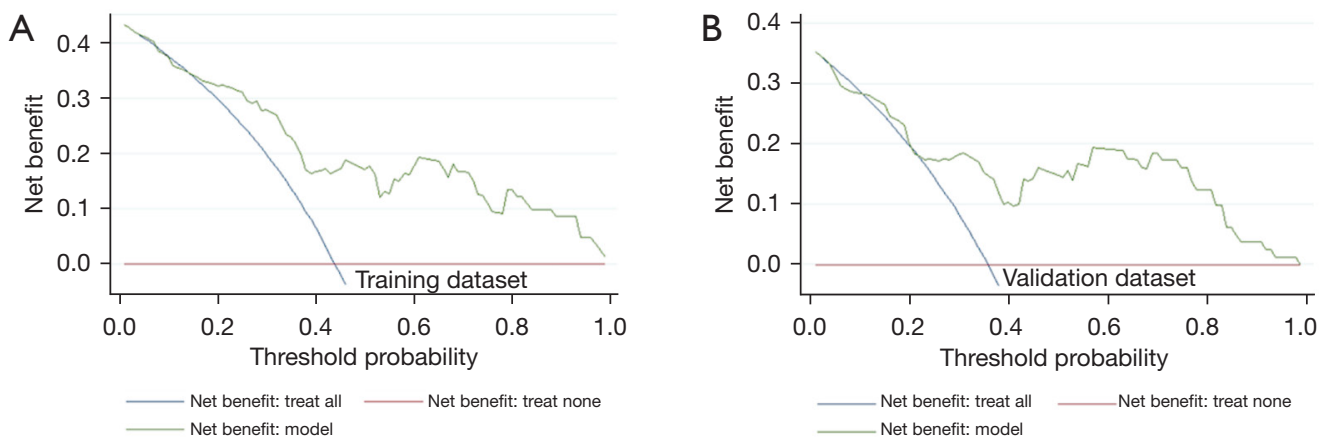

Figure 5 Decision curve analysis. The horizontal solid red line represents the assumption that no patients will experience bone metastasis, and the solid blue line represents the assumption that all patients will experience bone metastasis.

Comprehensive Cancer Network (NCCN) pointed out that T1 stage PCa patients with TPSA $>20 \mathrm{ng} / \mathrm{mL}$ were eligible for whole-bone scans (22). The American Urological Association (AUA) indicated that patients with localized PCa should have a whole bone scan if TPSA $>20 \mathrm{ng} / \mathrm{mL}$ (23). In this study, whole bone scans were performed on $125 \mathrm{PCa}$ patients with TPSA $\geq 20 \mathrm{ng} / \mathrm{mL}$, and bone metastases were found in $45.6 \%$ (57/125). Therefore, only using TPSA as a reference for bone scanning would cause a waste of medical resources. Therefore, this study attempted to use multiple high-risk factors for $\mathrm{PCa}$ bone metastasis to predict bone metastasis risk and confirm whether it is more effective than a single index.

In this study, ALP was divided into $<100,100-150$, and $>150 \mathrm{U} / \mathrm{L}$. As the ALP level increased, the proportion of bone metastasis patients increased. The risk of bone metastases in PCa patients increased by 1.839 times when ALP increased by $50 \mathrm{U} / \mathrm{L}$. Previous studies have also shown that high ALP is often accompanied by a high bone metastasis risk (24). Chen et al. (25) found that ALP can be used as a grouping standard at $120 \mathrm{U} / \mathrm{L}$, combined with TPSA, Gleason, and clinical tumor stage, to construct a risk assessment model for bone metastasis suitable for the Chinese population. In this study, 35 patients with bone metastases had a Gleason score of $\geq 8$, accounting for $53.8 \%$ of all patients with bone metastases. As the Gleason score increased, the proportion of patients with $\mathrm{PCa}$ with bone metastases increased. Chen et al. (19) showed that the proportion of bone scan positivity in PCa patients with Gleason $\geq 8$ points increased; in 45 patients with $\mathrm{PCa}$ with bone metastases, there were as many as $75.6 \%(34 / 45)$ patients with a Gleason score $\geq 8$; of the 35 patients without bone metastases, only six had a Gleason score $\geq 8$ (19). Nevertheless, the Gleason score in this study was not an independent predictor of PCa bone metastasis. Li et al. (26) also found that low serum testosterone and prostate puncture Gleason scores could not be used as independent factors to predict PCa bone metastasis risk. Serum ALP and TPSA can effectively predict bone metastasis of PCa. The Gleason score reported by the previous study is not the same for predicting the accuracy of $\mathrm{PCa}$ with bone metastasis. In practice, the clinical outcomes of $\mathrm{PCa}$ with the same Gleason score and similar histological type are often different. When the Gleason score of $\geq 8$ is used as the cut-off value for predicting bone metastasis of $\mathrm{PCa}$, the sensitivity and specificity are only $54.2 \%$ and $71.5 \%$, and the area under the ROC curve is only 0.68 (27). In a patient with PCa bone metastases after radical prostatectomy, the Gleason sum in prostatectomy specimens was not associated with bone scan positivity. TPSA elevation after radical prostatectomy is a more important predictor of bone metastasis progression than the preoperative Gleason score (28). Although Gleason is often used to predict patient outcomes, Gleason grades can also be heterogeneous in or between different tumor foci due to the presence of multifocal lesions in PCa. The value of combining other indicators to predict $\mathrm{PCa}$ bone metastases remains to be further studied.

A nomogram is a model tool that combines clinical outcomes with relevant factors. By adding the corresponding scores of related factors, the probability of occurrence of clinical outcomes can be obtained, and the probability of occurrence of the outcomes can be predicted for individuals, which has been applied to various diseases such as breast cancer, thyroid cancer, and PCa (29-31). In this study, the parameters of ultrasound contrast were explored. Combined with clinical indicators such as TPSA and ALP, a simple prediction model of bone metastasis of PCa was established to predict the risk of bone metastasis 
in patients with PCa. High-risk PCa can be found in the "first stop". When patients with a predicted high risk are found, attention should be paid to the occurrence of bone metastases, and a whole bone scan should be recommended. When the predicted value is lower, it is recommended that the doctor make a comprehensive judgment in combination with other imaging examinations and clinical indicators and perform a whole-bone scan if necessary. Although this model has a high positive predictive value because $\mathrm{PCa}$ is a heterogeneous tumor, when the predicted risk value is $\leq 25.0 \%$, the model still has a missed diagnosis rate of $8.7-12.2 \%$. Therefore, in future research, it is necessary to explore the combination of other indicators and imaging methods to reduce the missed diagnosis rate of the model.

Our model had a high degree of discrimination in both the training dataset and the validation dataset. The AUCs were 0.804 and 0.799 , respectively. The validation dataset also passed the calibration test, indicating that the model has a high degree of discrimination and better consistency. Besides, the decision analysis curve in this study showed that the model benefits patients in actual clinical diagnosis and treatment.

This study has limitations. This study was a single-center retrospective study performed at a tertiary reference hospital for PCa. A selection bias is inevitable, leading to $60.1 \%$ of the patients having total prostate-specific antigen (TPSA) levels $>30 \mathrm{ng} / \mathrm{mL}$. In addition, the number of patients in the bone metastasis group was small. The results require a multicenter large-sample randomized study for validation.

\section{Conclusions}

The TR-CEUS parameter AUC and clinical parameters TPSA and ALP are independent risk factors for predicting bone metastasis of PCa. The model established using these three factors can be used for an individualized assessment of the risk of bone metastasis in PCa patients.

\section{Acknowledgments}

Funding: The present study was supported by grants from the Key technology research plan project of the Inner Mongolia Autonomous Region (No. 2019GG096, No. 2019GG101).

\section{Footnote}

Conflicts of Interest: All authors have completed the ICMJE uniform disclosure form (available at https://dx.doi. org/10.21037/qims-21-365). All authors report that the present study was supported by grants from the Key technology research plan project of the Inner Mongolia Autonomous Region (No. 2019GG096, No. 2019GG101).

Ethical Statement: The authors are accountable for all aspects of the work in ensuring that questions related to the accuracy or integrity of any part of the work are appropriately investigated and resolved. The study was conducted in accordance with the Declaration of Helsinki (as revised in 2013). The study was approved by the ethics committee of the Inner Mongolia Autonomous Region People's Hospital, and individual consent for this retrospective analysis was waived.

Open Access Statement: This is an Open Access article distributed in accordance with the Creative Commons Attribution-NonCommercial-NoDerivs 4.0 International License (CC BY-NC-ND 4.0), which permits the noncommercial replication and distribution of the article with the strict proviso that no changes or edits are made and the original work is properly cited (including links to both the formal publication through the relevant DOI and the license). See: https://creativecommons.org/licenses/by-nc-nd/4.0/.

\section{References}

1. Siegel RL, Miller KD, Jemal A. Cancer statistics, 2018. CA Cancer J Clin 2018;68:7-30.

2. Bray F, Ferlay J, Soerjomataram I, Siegel RL, Torre LA, Jemal A. Global cancer statistics 2018: GLOBOCAN estimates of incidence and mortality worldwide for 36 cancers in 185 countries. CA Cancer J Clin 2018;68:394-424.

3. Chen $\mathrm{W}$, Zheng R, Baade PD, Zhang S, Zeng H, Bray F, Jemal A, Yu XQ, He J. Cancer statistics in China, 2015. CA Cancer J Clin 2016;66:115-32.

4. Harvey CJ, Pilcher J, Richenberg J, Patel U, Frauscher F. Applications of transrectal ultrasound in prostate cancer. Br J Radiol 2012;85 Spec No 1:S3-17.

5. Sturge J, Caley MP, Waxman J. Bone metastasis in prostate cancer: emerging therapeutic strategies. Nat Rev Clin Oncol 2011;8:357-68.

6. Sheikhbahaei S, Jones KM, Werner RA, Salas-Fragomeni RA, Marcus CV, Higuchi T, Rowe SP, Solnes LB, Javadi MS. 18F-NaF-PET/CT for the detection of bone metastasis in prostate cancer: a meta-analysis of diagnostic 
accuracy studies. Ann Nucl Med 2019;33:351-61.

7. Siegel C. Re: Transition Zone Prostate Cancer: Revisiting the Role of Multiparametric MRI at $3 \mathrm{~T}$. J Urol 2015;194:116.

8. Domachevsky L, Goldberg N, Gorenberg M, Bernstine H, Groshar D, Catalano OA. Prostate cancer evaluation using PET quantification in 68Ga-PSMA-11 PET/MR with attenuation correction of bones as a fifth compartment. Quant Imaging Med Surg 2020;10:40-7.

9. Roland A, Drouet C, Boulahdour H, Cochet A, De Bari B. Unusual uptakes on $18 \mathrm{~F}$-fluorocholine positron emission tomography/computed tomography (PET/CT): a retrospective study of 368 prostate cancer patients referred for a biochemical recurrence or an initial staging. Quant Imaging Med Surg 2021;11:172-82.

10. Cook GJ, Azad G, Padhani AR. Bone imaging in prostate cancer: the evolving roles of nuclear medicine and radiology. Clin Transl Imaging 2016;4:439-47.

11. Saini S. PSA and beyond: alternative prostate cancer biomarkers. Cell Oncol (Dordr) 2016;39:97-106.

12. Sano F, Uemura H. The utility and limitations of contrastenhanced ultrasound for the diagnosis and treatment of prostate cancer. Sensors (Basel) 2015;15:4947-57.

13. Liu G, Wu S, Huang L. Contrast-enhanced ultrasound evaluation of the prostate before transrectal ultrasoundguided biopsy can improve diagnostic sensitivity: A STARD-compliant article. Medicine (Baltimore) 2020;99:e19946.

14. Nicholson B, Theodorescu D. Angiogenesis and prostate cancer tumor growth. J Cell Biochem 2004;91:125-50.

15. Russo G, Mischi M, Scheepens W, De la Rosette JJ, Wijkstra H. Angiogenesis in prostate cancer: onset, progression and imaging. BJU Int 2012;110:E794-808.

16. Fillon M. Contrast-enhanced ultrasound may aid prostate cancer detection. J Natl Cancer Inst 2013;105:444-6.

17. Humphrey PA, Moch H, Cubilla AL, Ulbright TM, Reuter VE. The 2016 WHO Classification of Tumours of the Urinary System and Male Genital Organs-Part B: Prostate and Bladder Tumours. Eur Urol 2016;70:106-19.

18. Hanley JA, McNeil BJ. A method of comparing the areas under receiver operating characteristic curves derived from the same cases. Radiology 1983;148:839-43.

19. Chen Y, Lin Y, Nie P, Jiang W, Liu Y, Yuan R, Li M, Zhao S, Lin H, Li P, Zhang J, Hu Z, Xu J, Zhu X. Associations of Prostate-Specific Antigen, Prostate Carcinoma Tissue Gleason Score, and Androgen Receptor Expression with Bone Metastasis in Patients with Prostate Carcinoma. Med Sci Monit 2017;23:1768-74.
20. Yonou H, Aoyagi Y, Kanomata N, Kamijo T, Oda T, Yokose T, Hasebe T, Nagai K, Hatano T, Ogawa Y, Ochiai A. Prostate-specific antigen induces osteoplastic changes by an autonomous mechanism. Biochem Biophys Res Commun 2001;289:1082-7.

21. Briganti A, Passoni N, Ferrari M, Capitanio U, Suardi N, Gallina A, Da Pozzo LF, Picchio M, Di Girolamo V, Salonia A, Gianolli L, Messa C, Rigatti P, Montorsi F. When to perform bone scan in patients with newly diagnosed prostate cancer: external validation of the currently available guidelines and proposal of a novel risk stratification tool. Eur Urol 2010;57:551-8.

22. Kawachi MH, Bahnson RR, Barry M, Busby JE, Carroll PR, Carter HB, et al. NCCN clinical practice guidelines in oncology: prostate cancer early detection. J Natl Compr Canc Netw 2010;8:240-62.

23. Greene KL, Albertsen PC, Babaian RJ, Carter HB, Gann PH, Han M, Kuban DA, Sartor AO, Stanford JL, Zietman A, Carroll P; American Urological Association. Prostate specific antigen best practice statement: 2009 update. J Urol 2013;189:S2-S11.

24. Kataoka A, Yuasa T, Kageyama S, Tsuchiya N, Habuchi T, Iwaki H, Narita M, Okada Y, Yoshiki T. Diagnosis of bone metastasis in men with prostate cancer by measurement of serum ICTP in combination with alkali phosphatase and prostate-specific antigen. Clin Oncol (R Coll Radiol) 2006;18:480-4.

25. Chen S, Wang L, Qian K, Jiang W, Deng H, Zhou Q, Wang G, Liu X, Wu CL, Xiao Y, Wang X. Establishing a prediction model for prostate cancer bone metastasis. Int J Biol Sci 2019;15:208-20.

26. Li XB, Zhang L, Rao TW, Chen J, Leng YJ, Huang P. Low serum testosterone level does not predict bone metastasis of prostate cancer. Zhonghua Nan Ke Xue 2017;23:212-6.

27. Amoako YA, Hammond ENB, Assasie-Gyimah A, Laryea DO, Ankrah A, Amoah G. Prostate-specific antigen and risk of bone metastases in west Africans with prostate cancer. World J Nucl Med 2019;18:143-8.

28. Dotan ZA, Bianco FJ Jr, Rabbani F, Eastham JA, Fearn P, Scher HI, Kelly KW, Chen HN, Schöder H, Hricak H, Scardino PT, Kattan MW. Pattern of prostate-specific antigen (PSA) failure dictates the probability of a positive bone scan in patients with an increasing PSA after radical prostatectomy. J Clin Oncol 2005;23:1962-8.

29. Luo WQ, Huang QX, Huang XW, Hu HT, Zeng FQ, Wang W. Predicting Breast Cancer in Breast Imaging Reporting and Data System (BI-RADS) Ultrasound 
Category 4 or 5 Lesions: A Nomogram Combining Radiomics and BI-RADS. Sci Rep 2019;9:11921.

30. Öcal B, Korkmaz MH, Yılmazer D, Taşkın Türkmenoğlu T, Bayır Ö, Saylam G, Çadallı Tatar E, Karahan S, Çakal E. The Malignancy Risk Assessment of Cytologically Indeterminate Thyroid Nodules Improves Markedly by Using a Predictive Model. Eur Thyroid J 2019;8:83-9.

Cite this article as: Hong H, Liang D, Liu Q, Wu G, Sun R, Liu J, Wang F, Wang F. Value of transrectal contrastenhanced ultrasound with clinical indicators in the prediction of bone metastasis in prostate cancer. Quant Imaging Med Surg 2022;12(3):1750-1761. doi: 10.21037/qims-21-365
31. Ding Z, Song D, Wu H, Tian H, Ye X, Liang W, Xu J, Dong F. Development and validation of a nomogram based on multiparametric magnetic resonance imaging and elastography-derived data for the stratification of patients with prostate cancer. Quant Imaging Med Surg 2021;11:3252-62. 


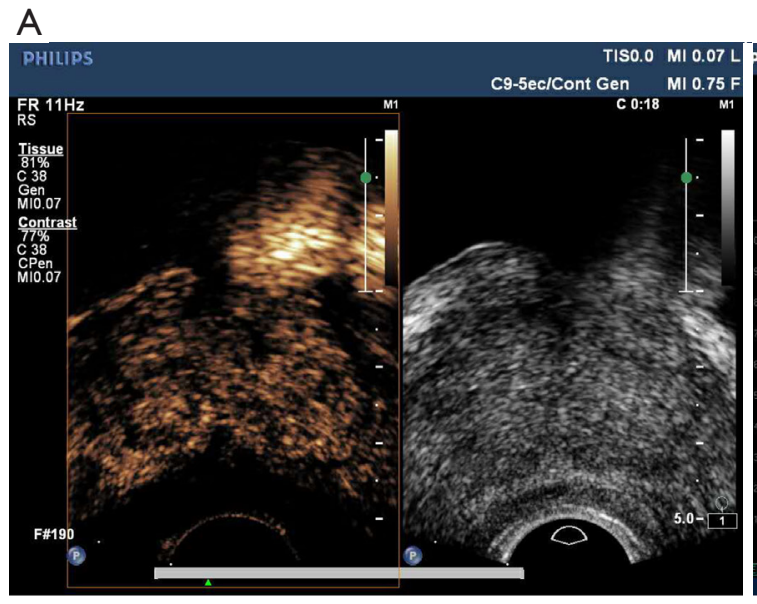

B
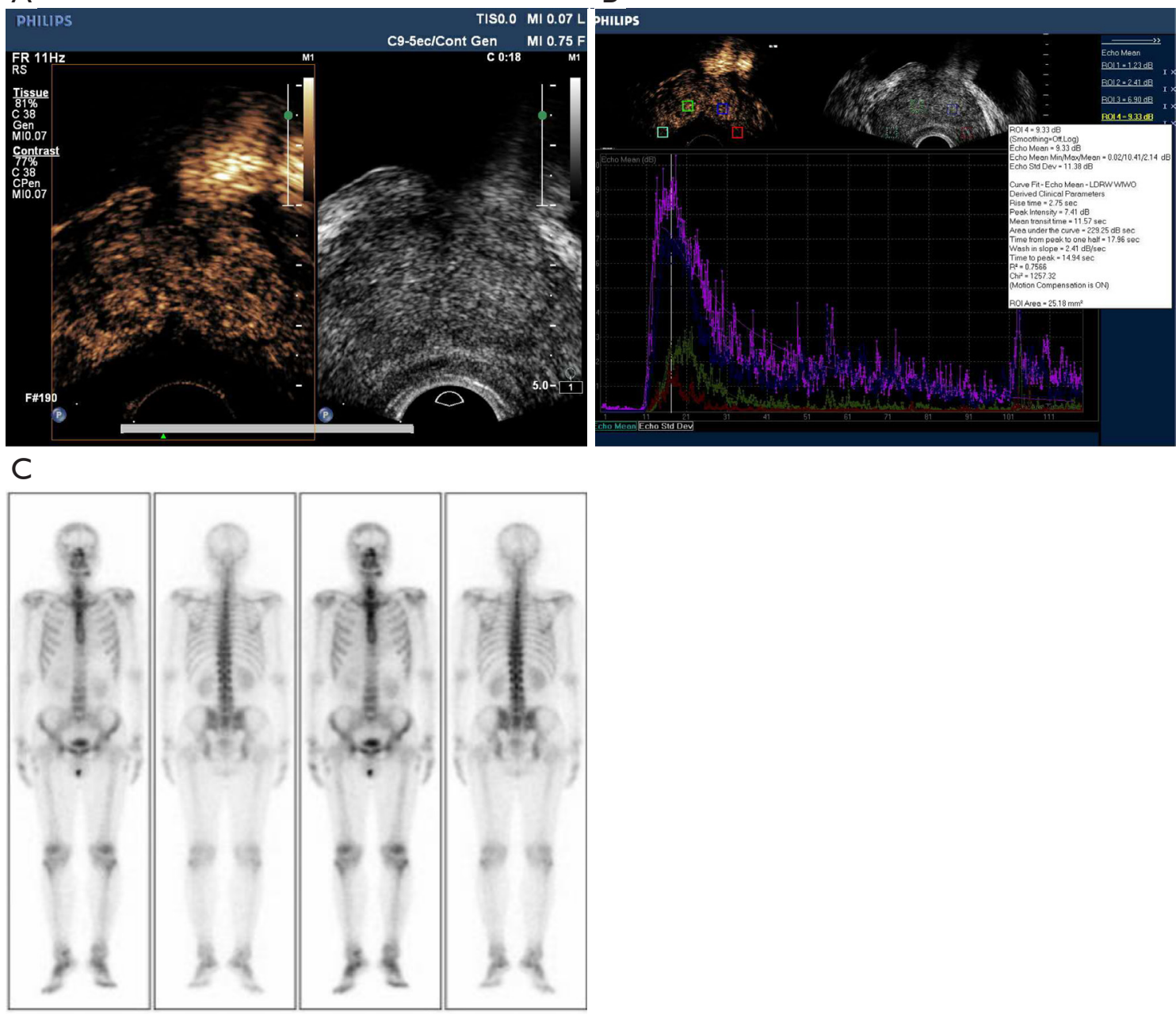

Figure S1 Patient with prostate cancer, 59 years old, with TPSA at $17.9 \mathrm{ng} / \mathrm{mL}$, ALP at $115.8 \mathrm{U} / \mathrm{L}$, and pathological Gleason score $4+3=7$ points. (A) TR-CEUS showed diffuse low enhancement of the prostate. (B) Analysis using the QLAB software in the prostate showed RT of $2.75 \mathrm{~s}$, PI of $7.41 \mathrm{~dB}, \mathrm{MTT}$ of $11.57 \mathrm{~s}$, AUC of $229.25 \mathrm{dBs}$, HT of $17.96 \mathrm{~s}$, WIS of $2.41 \mathrm{~dB} / \mathrm{s}$, and TTP of $14.94 \mathrm{~s}$. The bone metastasis model had a score of 48 points, a bone metastasis risk of $46 \%$, and a low risk of bone metastases. (C) A whole-bone scan was performed $3 \mathrm{~h}$ after intravenous injection of 99mTc-MDP. The scan included front and rear image acquisition. The whole bone scan image was clear and symmetrical, and no suspicion of metastasis was observed. TPSA, total prostate-specific antigen; ALP, alkaline phosphatase; TR-CEUS, Transrectal contrast-enhanced ultrasound; RT, rise time; PI, peak intensity; MTT, mean transit time; AUC, area under the curve; HT, intensity from peak to one-half time; WIS, wash-in slope; TTP, time to peak. 


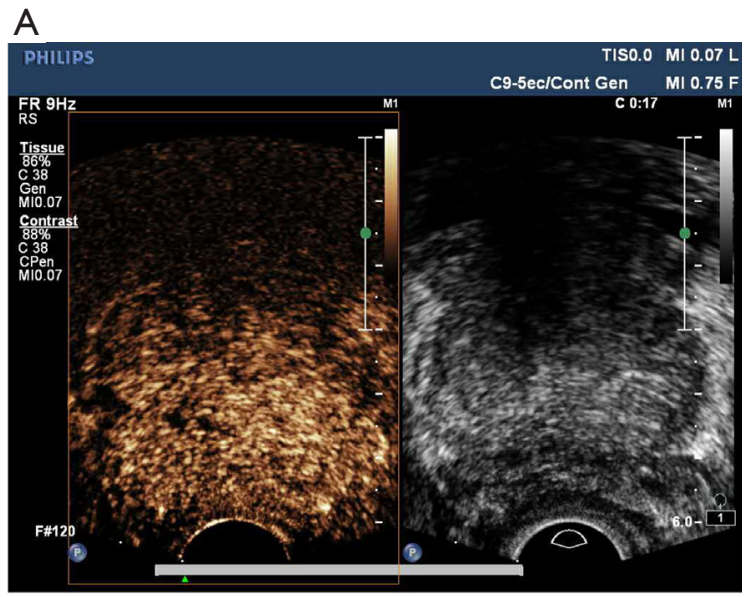

B
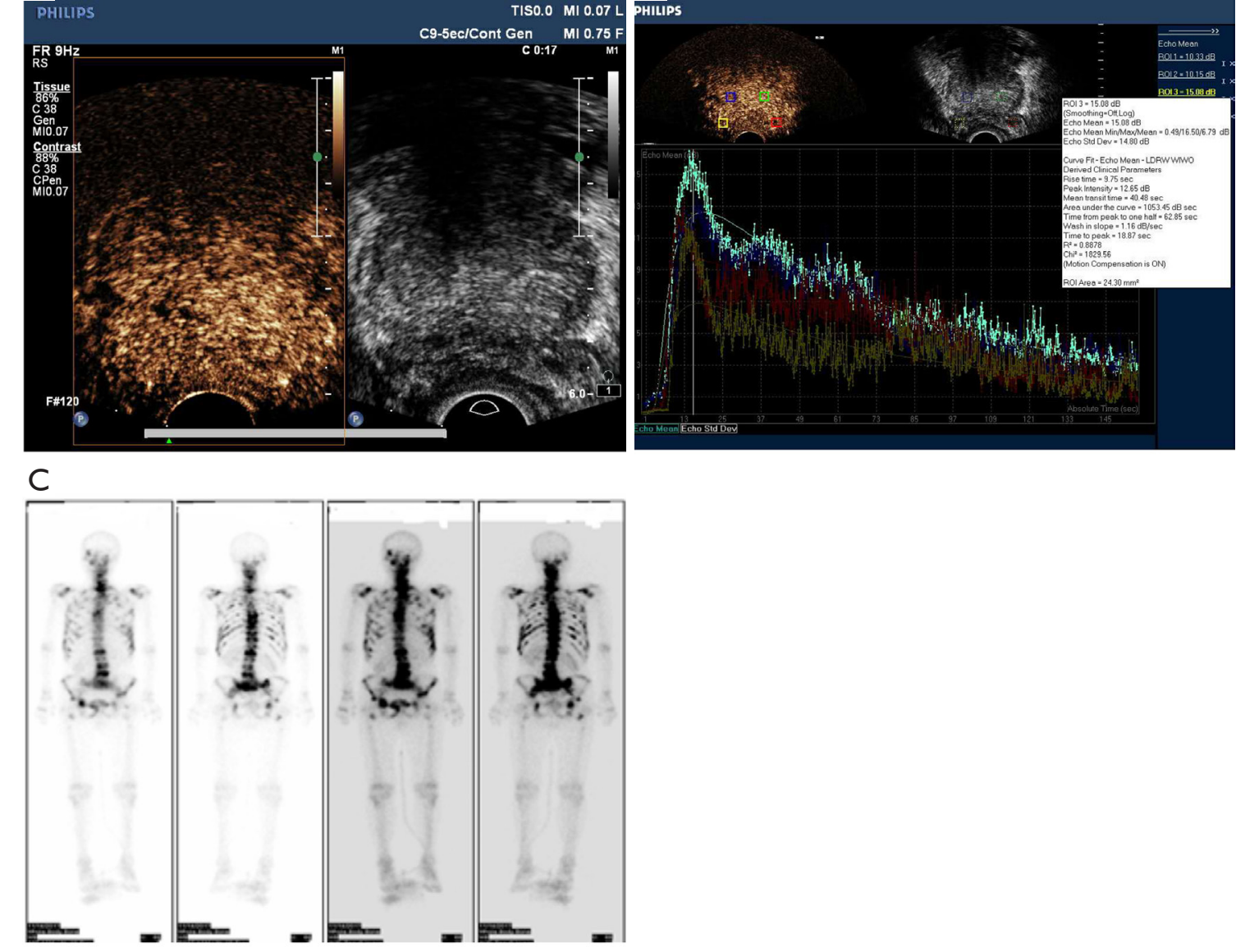

Figure S2 Patient with prostate cancer, 67 years old, with TPSA of $44.74 \mathrm{ng} / \mathrm{mL}$, ALP of $121.7 \mathrm{U} / \mathrm{L}$, and pathological Gleason score $5+5=10$ points. (A) TR-CEUS showed diffuse high enhancement of the prostate. (B) Analysis using the QLAB software in the prostate showed RT of $9.75 \mathrm{~s}$, PI of $12.65 \mathrm{~dB}, \mathrm{MTT}$ of $40.48 \mathrm{~s}$, AUC of $1,053.45 \mathrm{dBs}$, HT of $62.85 \mathrm{~s}$, WIS of $1.16 \mathrm{~dB} / \mathrm{s}$, and TTP of $18.87 \mathrm{~s}$. The bone metastasis model had a score of 110 points and a bone metastasis risk of $>90 \%$. The risk of bone metastasis was high. (C) A whole-bone scan was performed $3 \mathrm{~h}$ after intravenous injection of 99mTc-MDP. The scan included front and rear image acquisition. The whole bone scan image was clear, bilaterally symmetrical. There were multiple radioactive abnormalities in the skull base, sternum, bilateral ribs, spine, pelvis, and proximal femur. TPSA, total prostate-specific antigen; ALP, alkaline phosphatase; TR-CEUS, Transrectal contrast-enhanced ultrasound; RT, rise time; PI, peak intensity; MTT, mean transit time; AUC, area under the curve; HT, intensity from peak to one-half time; WIS, wash-in slope; TTP, time to peak. 
Table S1 Analysis of bone metastasis in the training and validation sets

\begin{tabular}{|c|c|c|c|c|c|c|}
\hline \multirow{2}{*}{ Indicators } & \multicolumn{3}{|c|}{ Training set $(n=82)$} & \multicolumn{3}{|c|}{ Validation dataset $(n=81)$} \\
\hline & Non-bone metastasis & Bone metastasis & $\mathrm{P}$ & Non-bone metastasis & Bone metastasis & $\mathrm{P}$ \\
\hline Age (years) & $72.5 \pm 7.9$ & $71.7 \pm 8.7$ & 0.643 & $70.4 \pm 6.5$ & $73.7 \pm 6.7$ & 0.035 \\
\hline Volume (mL) & $39.4(33.2)$ & $51.6(28.4)$ & 0.077 & $55.6(34.5)$ & $47.2(44.6)$ & 0.084 \\
\hline TPSA (ng/mL) & & & 0.007 & & & 0.384 \\
\hline$<20$ & $13(28.3)$ & $1(2.8)$ & & $17(32.7)$ & $7(24.1)$ & \\
\hline $20-30$ & 7 (15.2) & $5(13.9)$ & & $11(21.2)$ & $4(13.8)$ & \\
\hline$>30$ & $26(56.5)$ & $30(83.3)$ & & $24(46.2)$ & $18(62.1)$ & \\
\hline ALP (U/L) & & & $<0.001$ & & & $<0.001$ \\
\hline$<100$ & $38(82.6)$ & $22(61.1)$ & & $46(88.5)$ & $10(34.5)$ & \\
\hline $100-150$ & $8(17.4)$ & $3(8.3)$ & & $6(11.5)$ & $8(27.6)$ & \\
\hline$>150$ & $0(0.0)$ & $11(30.6)$ & & $0(0.0)$ & $8(27.6)$ & \\
\hline Gleason & & & 0.458 & & & 0.130 \\
\hline$\leq 6$ & $8(17.4)$ & $5(13.9)$ & & $16(30.8)$ & $3(10.3)$ & \\
\hline $3+4$ & $7(15.2)$ & 5 (13.9) & & $5(9.6)$ & $3(10.3)$ & \\
\hline $4+3$ & $17(37.0)$ & $8(22.2)$ & & $14(26.9)$ & $6(20.7)$ & \\
\hline 8 & $9(19.6)$ & $11(30.6)$ & & $8(15.4)$ & $10(34.5)$ & \\
\hline $9-10$ & 5 (10.9) & 7 (19.4) & & 9 (17.3) & 7 (24.1) & \\
\hline $\mathrm{PI}$ & $6.61(3.59)$ & $7.98(5.15)$ & 0.024 & $7.63(2.59)$ & $7.88(3.52)$ & 0.347 \\
\hline MTT & $25.25(14.52)$ & $28.15(10.44)$ & 0.230 & $25.34(9.73)$ & $26.06(17.00)$ & 0.961 \\
\hline $\mathrm{HT}$ & 37.08 (19.94) & $40.99(16.5)$ & 0.372 & $36.52(11.33)$ & 38.37 (23.29) & 0.902 \\
\hline WIS & $1.3(0.87)$ & $1.13(1.18)$ & 0.644 & $1.46(0.82)$ & $0.87(0.73)$ & 0.009 \\
\hline TTP & $25.48(8.91)$ & $24.57(7.74)$ & 0.667 & $23.84(9.32)$ & $25.1(10.23)$ & 0.879 \\
\hline $\mathrm{RT}$ & $6.84(3.27)$ & $6.84(3.54)$ & 0.837 & $6.29(3.13)$ & $7.27(4.53)$ & 0.157 \\
\hline AUC & 390.74 (232.04) & 485.61 (423.64) & 0.010 & 404.66 (190.00) & $410.67(285.4)$ & 0.146 \\
\hline
\end{tabular}

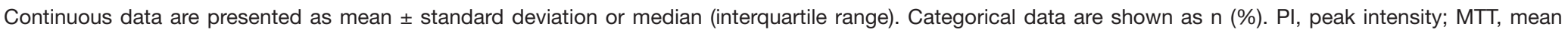

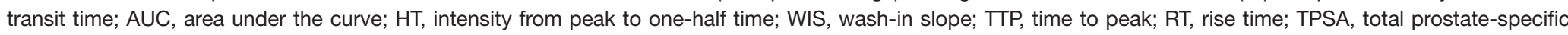
antigen; ALP, alkaline phosphatase. 
Table S2 ROC curve analysis of individual indicators in the training and validation sets

\begin{tabular}{|c|c|c|c|c|c|c|c|c|c|c|c|c|c|}
\hline \multirow[b]{2}{*}{ Variable } & \multicolumn{4}{|c|}{ Training set $(n=115)$} & \multicolumn{4}{|c|}{ Validation dataset $(n=48)$} & \multicolumn{5}{|c|}{ All $(n=163)$} \\
\hline & $\begin{array}{c}\text { AUC } \\
(95 \% \mathrm{Cl})\end{array}$ & Sensitivity & Specificity & Youden & $\begin{array}{c}\text { AUC } \\
(95 \% \mathrm{Cl})\end{array}$ & Sensitivity & Specificity & Youden & $\begin{array}{c}\text { AUC } \\
(95 \% \mathrm{Cl})\end{array}$ & Sensitivity & Specificity & Youden & $\mathrm{P}^{*}$ \\
\hline $\mathrm{PI}$ & $\begin{array}{c}0.647 \\
(0.553-0.734)\end{array}$ & 65.31 & 60.61 & 0.259 & $\begin{array}{c}0.502 \\
(0.354-0.650)\end{array}$ & 62.50 & 18.75 & 0.188 & $\begin{array}{c}0.606 \\
(0.527-0.682)\end{array}$ & 26.2 & 95.9 & 0.221 & $<0.001$ \\
\hline AUC & $\begin{array}{c}0.680 \\
(0.587-0.764)\end{array}$ & 42.86 & 98.48 & 0.413 & $\begin{array}{c}0.510 \\
(0.361-0.657)\end{array}$ & 56.25 & 25.00 & 0.188 & $\begin{array}{c}0.636 \\
(0.557-0.710)\end{array}$ & 36.9 & 98.0 & 0.349 & $<0.001$ \\
\hline Gleason & $\begin{array}{c}0.609 \\
(0.514-0.699)\end{array}$ & 51.02 & 71.21 & 0.222 & $\begin{array}{c}0.632 \\
(0.480-0.766)\end{array}$ & 62.60 & 62.50 & 0.250 & $\begin{array}{c}0.612 \\
(0.533-0.687)\end{array}$ & 53.9 & 68.4 & 0.222 & $<0.001$ \\
\hline ALP & $\begin{array}{c}0.672 \\
(0.578-0.757)\end{array}$ & 30.61 & 100.00 & 0.306 & $\begin{array}{c}0.809 \\
(0.669-0.908)\end{array}$ & 68.75 & 87.50 & 0.563 & $\begin{array}{c}0.707 \\
(0.630-0.775)\end{array}$ & 50.8 & 85.7 & 0.365 & 0.006 \\
\hline TPSA & $\begin{array}{c}0.620 \\
(0.524-0.709)\end{array}$ & 75.51 & 45.45 & 0.21 & $\begin{array}{c}0.613 \\
(0.705-0.930)\end{array}$ & 68.75 & 56.25 & 0.250 & $\begin{array}{c}0.624 \\
(0.545-0.699)\end{array}$ & 73.9 & 49.0 & 0.228 & $<0.001$ \\
\hline
\end{tabular}

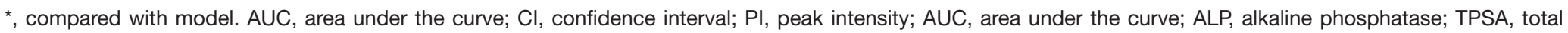
prostate-specific antigen. 